\title{
Consumo comunitario de antimicrobianos en Chile, 2000-2008
}

\author{
Luis Bavestrello F. y Ángela Cabello M.
}

\section{Community antibiotic consumption in Chile, 2000-2008}

Background: The Chilean Ministry of Health has implemented regulatory rules for the consumption of antibiotics since September 1999, with sales restriction limited only with medical prescription. Aim: To analyze the impact of established regulatory measures from 2000 to 2008. Material and Methods: A retrospective analysis of antibiotics sales in pharmacies from 2000 to 2008 was performed. The information was obtained from the International Marketing System (IMS Health), an auditing system of pharmacy sales. The consumption unit used was the Defined Daily Dose per 1000 inhabitants/day (DDD). Results: From 2000 to 2002 the regulatory rules had a great impact, but since 2002 the antibiotic consumption increased, especially amoxicillin, returning to similar levels observed in 1998. Conclusions: The regulatory measures had an initial impact, but there was not reinforcement in the time and there was no further control. It is necessary to assume a permanent task and support of the authorities of health to educate the population about the implications of the inadequate use of antimicrobials and his effect on the microbial ecology.

Key words: Antibiotics, regulatory rules, antibacterial resistance, prescription, consumption.

Palabras clave: Antimicrobianos, medidas regulatorias, resistencia bacteriana, receta médica, consumo.

\section{Introducción}

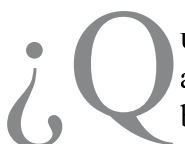
ué ha ocurrido en Chile en los últimos 10 años, respecto del consumo de antimicrobianos, después del éxito inicial de las medidas regulatorias? Esta es la interrogante que el presente artículo quiere contestar, siguiendo la misma modalidad utilizada en estudios precedentes ${ }^{1-3}$. En ellos, se presenta desde 1988 la realidad de consumo comunitario, lo que motivó en 1999 que el Ministerio de Salud de Chile aplicara medidas regulatorias a la venta de antimicrobianos en farmacias privadas, haciendo cumplir lo señalado en los rótulos de los envases "venta bajo receta médica". De esta forma, las autoridades de salud del país pretendían evitar la automedicación con este grupo de medicamentos y revertir el aumento de consumo progresivo documentado desde $1988^{1}$. Por esto, y debido a que pueden presentar reacciones adversas como todo fármaco, que su uso inadecuado va ligado a emergencia de resistencia bacteriana y constituye un gasto innecesario, se considera que el uso de estos fármacos debe ir respaldado por una prescripción médica ${ }^{4-6}$.

El impacto que tuvo la aplicación de las medidas fue publicado $^{3}$ y ampliamente difundido ${ }^{4,7,8}$. El consumo de la mayoría de antimicrobianos, expresado en DDD/1.000 habitantes-día, como cloranfenicol, cotrimoxazol, eritromicina, cloxacilina, ampicilina y amoxicilina, disminuyó significativamente, sin que esto se asociara a un aumento de la morbilidad infecciosa.

Estos estudios mostraron que las medidas regulatorias de 1999 fueron exitosas ya que lograron disminuir el consumo de antimicrobianos que se expendían por automedicación en las farmacias. Se eliminaron los antimicrobianos de las listas con incentivo a las ventas en las farmacias y se comunicó a la población que sin receta médica no obtendría antimicrobianos, lo que limitó las solicitudes. Ante la advertencia de fiscalización de las medidas, el cumplimiento de ellas fue efectivo.

Para mostrar lo que ha sucedido después, desde el año 2000 hasta el 2008, período en el cual no se tomaron nuevas medidas ni se reforzaron aquellas implementadas, usamos nuevamente la información proveniente del International Marketing System Health (IMS Health), empresa internacional dedicada a la auditoría de datos de venta farmacéutica ${ }^{9}$. La información obtenida corresponde sólo a la venta a público de unidades de envases de antimicrobianos orales en las farmacias privadas comerciales del país, quedando excluidos aquellos dispensados en los consultorios adosados a los hospitales públicos, privados y consultorios de atención primaria de salud, en los cuales, la dispensación de antimicrobianos se realiza siempre con prescripción médica.

Como unidad de medida de consumo se mantuvo la utilizada en estudios anteriore ${ }^{1-3}$ que evita el sesgo dado
Clínica Reñaca, Viña del Mar, Chile (LBF). Hospital Gustavo Fricke, Viña del Mar, Chile Farmacia (ACM).

Los autores declaran no tener conflictos de interés y no contar con fuente de financiamiento en la preparación de este artículo.

Recibido: 15 de julio de 2010 Aceptado: 23 de diciembre de 2010

Correspondencia a: Luis Bavestrello Fernández Ibavestrello@gmail.com 
por las unidades de envases y corresponde a la Dosis Diaria Definida por 1.000 habitantes por día (DDD/1.000 habitantes-día) que refleja el consumo de la Dosis Diaria Definida (DDD) específica del antimicrobiano respectivo por cada 1.000 habitantes en un día ${ }^{10,11}$. Dentro de las ventajas de utilizar esta unidad de medida, es que representa un indicador de consumo, permite efectuar comparaciones en diferentes períodos de tiempo, está recomendada por la OMS para estudios farmacoepidemiológicos, es de fácil acceso y bajo costo. Sus principales limitaciones son que la DDD específica no coincide necesariamente con la utilizada en clínica y que considera sólo dosificaciones de adultos.

\section{Materiales y Método}

El presente estudio, de tipo retrospectivo, utiliza la información obtenida a través de los datos de venta de las unidades de envases o cajas individuales de antimicrobianos orales desde los años 2000 al año 2008, información obtenida del IMS Health ${ }^{11}$.

Para ello, se reunieron los datos de las especialidades farmacéuticas vendidas de un mismo antimicrobiano, se separaron según nombre genérico y se reunieron en los siguientes grupos:

- $\beta$-lactámicos (amoxicilina, ampicilina, amoxicilina/ ácido clavulánico, amoxicilina/sulbactam, sultamicilina, cloxacilina, flucloxacilina, fenoximetilpenicilina y cefalosporinas orales).

- Macrólidos y similares (eritromicina, claritromicina, azitromicina, lincomicina, clindamicina, miocamicina, roxitromicina, telitromicina).

- Quinolonas (ciprofloxacina, levofloxacina, moxifloxacina, norfloxacina).

- Cotrimoxazol.

- Tetraciclinas y asociados (tetraciclina, doxiciclina, minociclina).

- Cloranfenicol.

Como se trata de consumo comunitario, se consideran sólo las presentaciones de uso oral, ya sea sólida, como comprimido o tableta, o líquida como suspensión o jarabe. Dado que la unidad de medida de consumo utilizada es la DDD/1.000 habitantes-día, fue necesario expresar las unidades de envases vendidos de antimicrobianos en gramos, para lo cual se consideró la concentración y cantidad de la forma de presentación que incluía, el cual se multiplicó por la cantidad vendida de dicha formulación. La suma de los totales vendidos de las diferentes formulaciones permitió obtener el total de gramos vendidos para un determinado antimicrobiano.

La fórmula utilizada fue:
DDD/1.000 habitantes-día $=$

gramos vendidos * 1.000

$\mathrm{N}^{\circ}$ días de estudio*DDD específica (11)*población

Los datos de población se refieren a población total del país en cada año y fueron obtenidos del Instituto Nacional de Estadística, INE, de Chile ${ }^{12}$ para cada año, las Dosis Diaria Definida específica (DDD específica) de cada antimicrobiano corresponden a las elaboradas por el Consejo Nórdico de Medicina ${ }^{11}$ y el número de días de estudio correspondió a un año (365 días). De esta forma se obtuvieron las DDD/1.000 habitantes-día para cada antimicrobiano.

\section{Resultados}

La Tabla 1 muestra los valores obtenidos de DDD/1.000 habitantes-día, desde el año 2000 al año 2008, para todos los antimicrobianos orales que se comercializaron y vendieron en el país. Al final de la Tabla 1 se aprecia la Sumatoria de DDD, para cada año, la cual se observa que aumenta paulatinamente hasta alcanzar un valor de 10.064 el año 2007. También se observa que el antimicroiano de mayor consumo fue amoxicilina.

La Tabla 2 muestra las DDD/1.000 habitantes-día de los antimicrobianos $\beta$-lactámicos orales, incluyendo aminopenicilinas, otras penicilinas como cloxacilina, flucloxacilina y fenoximetilpenicilina. También están incluidas aminopenicilinas con inhibidores de $\beta$-lactamasas y cefalosporinas orales. La primera columna muestra el año 1998, antes de la aplicación de las medidas regulatorias, a objeto de resaltar el impacto inicial de las medidas regulatorias. Pese a la disminución de consumo de ampicilina desde 1999, el grupo de aminopenicilinas lidera el consumo en el período estudiado.

La Figura 1 muestra la gráfica correspondiente a $\beta$-lactámicos y claramente se observa que el antimicrobiano más vendido y por consecuencia, consumido, corresponde a amoxicilina.

La Tabla 3 muestra los resultados obtenidos en DDD/1.000 habitantes-día para quinolonas, desde el año 2000 a 2008, donde se observa el aumento progresivo de consumo a través de los años, predominando ciprofloxacina, que en 1998 era el único representante del grupo.

En la Tabla 4 se observa el consumo de macrólidos, donde es claritromicina el antimicrobiano que predomina en ventas durante el período estudiado y eritromicina es el antimicrobiano que disminuye cada año su consumo. Dado que mientras azitromicina y claritromicina aumentan y eritromicina disminuye, el valor total de $\mathrm{DDD} / 1.000$ habitantes-día del grupo macrólidos no ha experimentado gran variación. Como referencia, se muestra en la primera columna el año 1998. 
Tabla 1. Consumo de antimicrobianos expresado en DDD/1.000 habitantes-día, obtenidos de información de venta en farmacias comerciales de Chile, entre los años 2000 y 2008 (Fuente IMS Health)

\begin{tabular}{|c|c|c|c|c|c|c|c|c|c|}
\hline Antimicrobianos & 2000 & 2001 & 2002 & 2003 & 2004 & 2005 & 2006 & 2007 & 2008 \\
\hline Amoxicilina & 3,092 & 3,877 & 3,278 & 3,338 & 3,861 & 4,174 & 3,902 & 4,555 & 4,589 \\
\hline Ampicilina & 0,134 & 0,133 & 0,091 & 0,073 & 0,070 & 0,058 & 0,051 & 0,057 & 0,074 \\
\hline Amoxicilina/ác. clavulánico & 0,501 & 0,571 & 0,534 & 0,590 & 0,732 & 0,721 & 0,757 & 0,908 & 0,829 \\
\hline Amoxicilina/sulbactam & 0,000 & 0,007 & 0,003 & 0,003 & 0,002 & 0,000 & 0,000 & 0,000 & 0,000 \\
\hline Claritromicina & 0,593 & 0,724 & 0,635 & 0,626 & 0,766 & 0,855 & 0,805 & 0,903 & 0,783 \\
\hline Azitromicina & 0,385 & 0,451 & 0,374 & 0,294 & 0,449 & 0,490 & 0,538 & 0,736 & 0,672 \\
\hline Eritromicina & 0,358 & 0,361 & 0,280 & 0,201 & 0,219 & 0,176 & 0,157 & 0,154 & 0,140 \\
\hline Telitromicina & 0,000 & 0,000 & 0,006 & 0,006 & 0,004 & 0,002 & 0,000 & 0,000 & 0,000 \\
\hline Miocamicina & 0,007 & 0,003 & 0,000 & 0,000 & 0,000 & 0,000 & 0,000 & 0,000 & 0,000 \\
\hline Roxitromicina & 0,010 & 0,008 & 0,005 & 0,006 & 0,006 & 0,006 & 0,005 & 0,003 & 0,003 \\
\hline Clindamicina & 0,007 & 0,007 & 0,007 & 0,007 & 0,008 & 0,008 & 0,008 & 0,008 & 0,006 \\
\hline Lincomicina & 0,059 & 0,058 & 0,049 & 0,044 & 0,042 & 0,035 & 0,031 & 0,029 & 0,027 \\
\hline Cloxacilina & 0,306 & 0,295 & 0,272 & 0,261 & 0,270 & 0,249 & 0,248 & 0,243 & 0,243 \\
\hline Flucloxacilina & 0,158 & 0,163 & 0,155 & 0,144 & 0,141 & 0,142 & 0,161 & 0,170 & 0,178 \\
\hline Fenoximetilpenicilina & 0,085 & 0,065 & 0,045 & 0,041 & 0,036 & 0,027 & 0,021 & 0,013 & 0,017 \\
\hline Sultamicilina & 0,007 & 0,003 & 0,002 & 0,002 & 0,002 & 0,003 & 0,002 & 0,000 & 0,000 \\
\hline Cefadroxilo & 0,147 & 0,160 & 0,152 & 0,155 & 0,171 & 0,171 & 0,175 & 0,199 & 0,209 \\
\hline Cefuroximo axetil & 0,030 & 0,037 & 0,078 & 0,077 & 0,070 & 0,061 & 0,055 & 0,053 & 0,047 \\
\hline Cefixima & 0,018 & 0,017 & 0,014 & 0,013 & 0,013 & 0,011 & 0,011 & 0,010 & 0,008 \\
\hline Cefradina & 0,035 & 0,040 & 0,036 & 0,032 & 0,032 & 0,030 & 0,028 & 0,029 & 0,027 \\
\hline Cefaclor & 0,005 & 0,004 & 0,002 & 0,000 & 0,000 & 0,000 & 0,000 & 0,000 & 0,000 \\
\hline Cefprozil & 0,009 & 0,008 & 0,006 & 0,003 & 0,001 & 0,000 & 0,000 & 0,000 & 0,000 \\
\hline Cefalexina & 0,001 & 0,001 & 0,001 & 0,001 & 0,001 & 0,000 & 0,002 & 0,002 & 0,002 \\
\hline Ciprofloxacina & 0,424 & 0,487 & 0,474 & 0,535 & 0,596 & 0,613 & 0,659 & 0,719 & 0,785 \\
\hline Moxifloxacina & 0,000 & 0,027 & 0,029 & 0,029 & 0,042 & 0,045 & 0,048 & 0,066 & 0,061 \\
\hline Levofloxacina & 0,050 & 0,122 & 0,119 & 0,177 & 0,259 & 0,266 & 0,274 & 0,321 & 0,274 \\
\hline Cefpodoxima & 0,000 & 0,000 & 0,000 & 0,000 & 0,011 & 0,014 & 0,036 & 0,036 & 0,035 \\
\hline Norfloxacina & 0,012 & 0,007 & 0,007 & 0,006 & 0,005 & 0,004 & 0,004 & 0,004 & 0,003 \\
\hline Cotrimoxazol & 0,544 & 0,444 & 0,327 & 0,336 & 0,339 & 0,272 & 0,252 & 0,246 & 0,202 \\
\hline Tetraciclina & 0,264 & 0,226 & 0,204 & 0,189 & 0,188 & 0,163 & 0,142 & 0,143 & 0,163 \\
\hline Doxiciclina & 0,331 & 0,330 & 0,311 & 0,291 & 0,315 & 0,333 & 0,395 & 0,456 & 0,465 \\
\hline Minociclina & 0,045 & 0,065 & 0,059 & 0,066 & 0,070 & 0,070 & 0,063 & 0,050 & 0,052 \\
\hline Cloranfenicol & 0,045 & 0,038 & 0,032 & 0,028 & 0,028 & 0,025 & 0,021 & 0,017 & 0,016 \\
\hline Sumatoria DDD & 7,662 & 8,739 & 7,587 & 7,574 & 8,749 & 9,024 & 8,851 & 10,064 & 9,910 \\
\hline
\end{tabular}

\begin{tabular}{|lllllllllll|l|}
\hline \multicolumn{10}{|c|}{ Tabla 2. DDD/1.000 habitantes-día de antimicrobianos $\beta$-lactámicos orales } \\
\hline Antimicrobianos & $\mathbf{1 9 9 8}$ & $\mathbf{2 0 0 0}$ & $\mathbf{2 0 0 1}$ & $\mathbf{2 0 0 2}$ & $\mathbf{2 0 0 3}$ & $\mathbf{2 0 0 4}$ & $\mathbf{2 0 0 5}$ & $\mathbf{2 0 0 6}$ & $\mathbf{2 0 0 7}$ & $\mathbf{2 0 0 8}$ \\
Amoxicilina & 5,57 & 3,092 & 3,877 & 3,278 & 3,338 & 3,861 & 4,174 & 3,902 & 4,555 & 4,589 \\
Ampicilina & 0,494 & 0,134 & 0,133 & 0,091 & 0,073 & 0,070 & 0,058 & 0,051 & 0,057 & 0,074 \\
Otras penicilinas orales & 0,932 & 0,549 & 0,523 & 0,472 & 0,446 & 0,447 & 0,418 & 0,430 & 0,426 & 0,438 \\
Amoxicilina /inhib. $\beta$-lactamasas & 0,528 & 0,501 & 0,578 & 0,537 & 0,593 & 0,734 & 0,721 & 0,757 & 0,908 & 0,829 \\
Cefalosporinas orales & 0,297 & 0,245 & 0,267 & 0,289 & 0,281 & 0,299 & 0,287 & 0,307 & 0,329 & 0,328 \\
Total DDD/1.000 habitantes-día & 7,821 & 4,521 & 5,378 & 4,667 & 4,731 & 5,411 & 5,658 & 5,447 & 6,275 & 6,258 \\
\hline
\end{tabular}


Figura 1. Vista gráfica de la variación de DDD/1.000 habitantes-día para antimicrobianos $\beta$-lactámicos, entre los años 2000 y 2008.

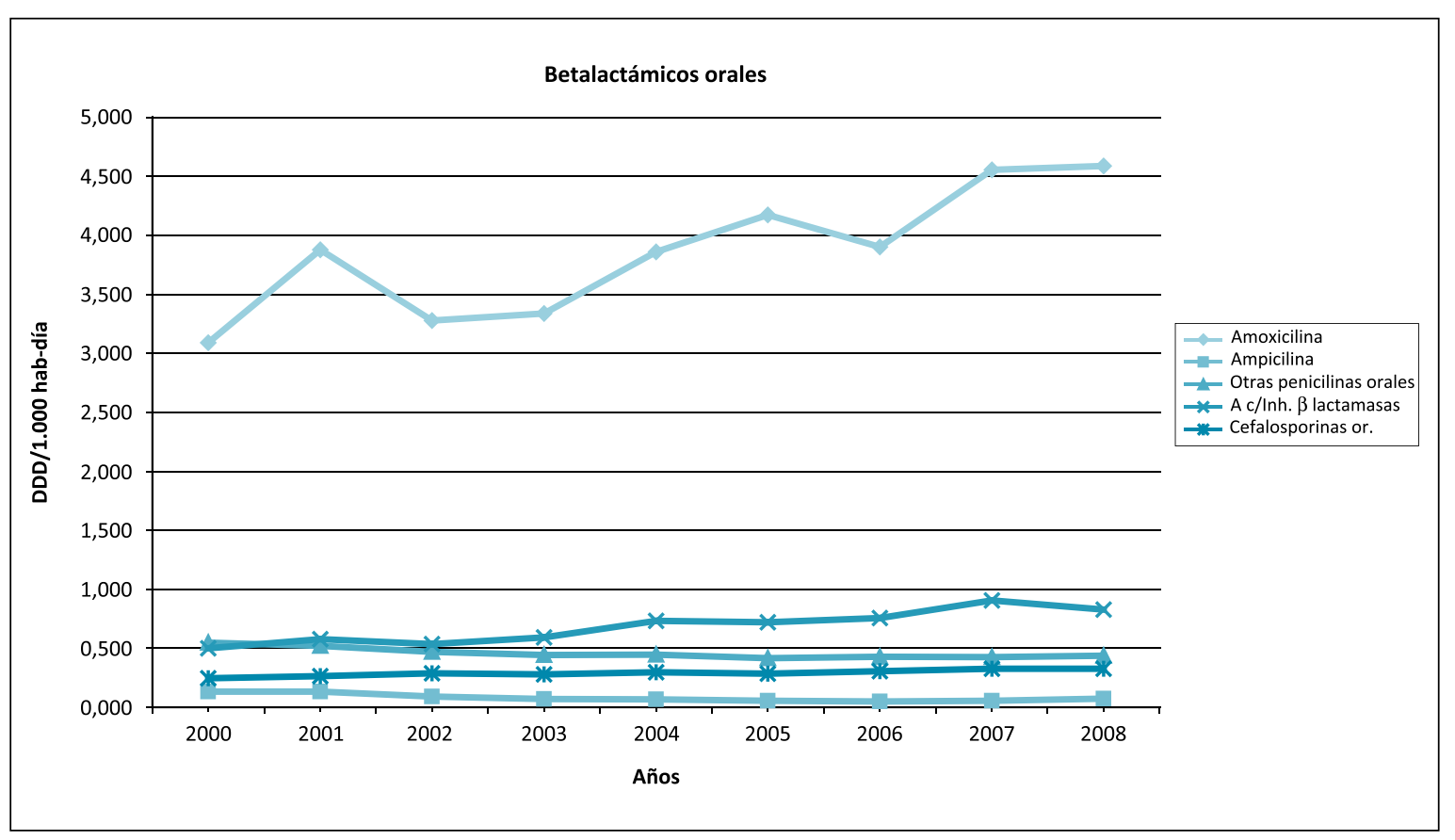

Tabla 3. Consumo de quinolonas expresado en DDD/1.000 habitantes-día

\begin{tabular}{|lcccccccccc|}
\hline Antimicrobianos & $\mathbf{1 9 9 8}$ & $\mathbf{2 0 0 0}$ & $\mathbf{2 0 0 1}$ & $\mathbf{2 0 0 2}$ & $\mathbf{2 0 0 3}$ & $\mathbf{2 0 0 4}$ & $\mathbf{2 0 0 5}$ & $\mathbf{2 0 0 6}$ & $\mathbf{2 0 0 7}$ & $\mathbf{2 0 0 8}$ \\
Norfloxacina & & 0,012 & 0,007 & 0,007 & 0,006 & 0,005 & 0,004 & 0,004 & 0,004 & 0,003 \\
Ciprofloxacina & 0,312 & 0,424 & 0,487 & 0,474 & 0,535 & 0,596 & 0,613 & 0,659 & 0,719 & 0,785 \\
Moxifloxacina & & 0,000 & 0,027 & 0,029 & 0,029 & 0,042 & 0,045 & 0,048 & 0.066 & 0,061 \\
Levofloxacina & & 0,050 & 0,122 & 0,119 & 0,177 & 0,259 & 0,266 & 0,274 & 0,321 & 0,274 \\
DDD/1.000 habitantes-día & 0,312 & 0,486 & 0,643 & 0,629 & 0,747 & 0,902 & 0,928 & 0,985 & 1,044 & 1,123 \\
\hline
\end{tabular}

Tabla 4. Consumo de macrólidos, expresado en DDD/1.000 habitantes-día

\begin{tabular}{|lllllllllll|}
\hline Antimicrobianos & $\mathbf{1 9 9 8}$ & $\mathbf{2 0 0 0}$ & $\mathbf{2 0 0 1}$ & $\mathbf{2 0 0 2}$ & $\mathbf{2 0 0 3}$ & $\mathbf{2 0 0 4}$ & $\mathbf{2 0 0 5}$ & $\mathbf{2 0 0 6}$ & $\mathbf{2 0 0 7}$ & $\mathbf{2 0 0 8}$ \\
Claritromicina & 0,41 & 0,593 & 0,724 & 0,635 & 0,626 & 0,766 & 0,855 & 0,805 & 0,903 & 0,783 \\
Azitromicina & 0,373 & 0,385 & 0,451 & 0,374 & 0,294 & 0,449 & 0,490 & 0,538 & 0,736 & 0,672 \\
Eritromicina & 0,63 & 0,358 & 0,361 & 0,280 & 0,201 & 0,219 & 0,176 & 0,157 & 0,154 & 0,140 \\
DDD/1.000 habitantes-día & 1,413 & 1,336 & 1,536 & 1,289 & 1,121 & 1,434 & 1,521 & 1,500 & 1,793 & 1,595 \\
\hline
\end{tabular}

\begin{tabular}{|lccccccccccc|}
\multicolumn{10}{c|}{ Tabla 5. Gasto en antimicrobianos orales vendidos en farmacias comerciales de Chile, } \\
expresados en \\
millones de dólares
\end{tabular}


La Tabla 5 resume el gasto, expresado en millones de dólares, involucrado en la compra de antimicrobianos orales por la población en Chile, entre los años 2000 y 2008. Se observa que hay una clara tendencia de aumento entre los años 2003 y 2008.

La Figura 2 muestra la sumatoria de DDD/1.000 habitantes-día para todos los antimicrobanos del presente estudio y permite apreciar que existe un aumento paulatino de consumo desde el año 2002 en adelante.

\section{Discusión}

La principal ventaja del uso de la medida de consumo $\mathrm{DDD} / 1.000$ habitantes-día es que permite efectuar comparaciones en diferentes períodos de tiempo. No es posible conocer el valor ideal de DDD/1.000 habitantes-día para cada antimicrobiano.Además, en Chile, el valor encontrado está subestimado debido a que sólo considera aquellas unidades de envases vendidas en farmacias privadas o comerciales y no considera lo entregado bajo prescripción médica en consultorios de salud, ya sea públicos y/o privados. Esto lleva a comparaciones equívocas con países europeos, donde las DDD/1.000 habitantes-día consideran a toda la población del país. La situación de Chile es igual al resto de países latinoamericanos, donde la $\mathrm{DDD} / 1.000$ habitantes-día es sólo una medida parcial de consumo.

A pesar de estas consideraciones, se observa en la Tabla 1 que amoxicilina continúa siendo el antimicrobiano más

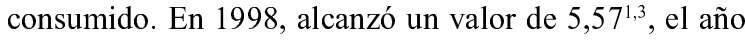
2000 disminuyó a 3,092, producto de las medidas regulatorias implementadas en septiembre de 1999 y el año 2008 tiene un valor de 4,859. Este antimicrobiano es el más vendido en todas sus formas farmacéuticas, lo que se refleja claramente en la Figura 1. Esta situación concuerda con un estudio realizado en Inglaterra ${ }^{13}$ donde amoxicilina superaba el $25 \%$ de todas las prescripciones de antimicrobianos, ocupando el primer lugar en los consumos.

También se observa en la Tabla 1 que las medidas regulatorias implementadas el año 1999 fueron exitosas para algunos antimicrobianos como ampicilina, eritromicina, cloxacilina, fenoximetilpenicilina, cloranfenicol, cotrimoxazol, y tetraciclina, cuyas DDD/1.000 habitantes-día bajaron abruptamente a partir de septiembre $1999^{3}$ y este descenso se ha mantenido en valores constantes entre los años 2000 y 2008.

La Tabla 2 que muestra, en conjunto, los $\beta$-lactámicos orales y la Figura 1 muestran una tendencia al aumento de consumo en el grupo de aminopenicilinas y aminopenicilinas con inhibidores de $\beta$-lactamasas. Sin embargo, en el segundo grupo, esta tendencia es leve aunque constante si la relacionamos con el consumo masivo descrito de amoxicilina. Las cefalosporinas orales han tenido un leve aumento debido al consumo creciente de cefadroxilo, que lidera el grupo. En un estudio realizado considerando el

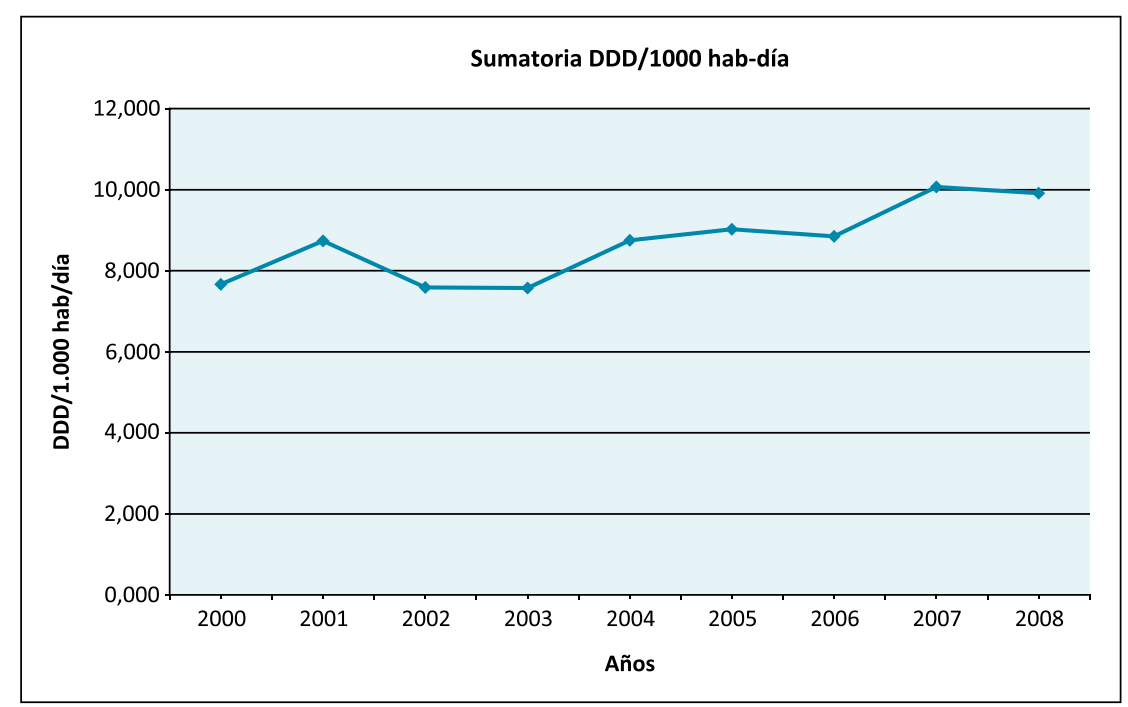

Figura 2. Gráfico de la sumatoria DDD/1.000 habitantes-día de antimicrobianos contenidos en la Tabla 1.

consumo de antimicrobianos en ocho países latinoamericanos se encontró que la clase terapéutica penicilinas lideraba el consumo. El consumo chileno de penicilinas sólo fue superado por Argentina ${ }^{15}$.

Existen un grupo de antimicrobianos que aumentó su consumo e incluso las medidas regulatorias no tuvieron impacto sobre él y corresponde a las quinolonas. El grupo de macrólidos experimentó un equilibrio ya que la disminución de consumo de eritromicina se compensó con el aumento de consumo de claritromicina y azitromicina. Esto puede reflejar que las medidas orientadas a la dispensación no tuvieron impacto en la prescripción, ya que estos grupos de antimicrobianos son mayoritariamente recetados por médicos. Ambos grupos pueden observarse en las Tablas 3 y 4 . La situación que ocurre con estos antimicrobianos coincide con un estudio de prescripción realizado en la comunidad en E.U.A., entre los años 1992 y 2000 , publicado el año $2003^{14}$ donde quinolonas, claritromicina, azitromicina, junto a aminopenicilinas con inhibidores de $\beta$-lactamasas también aumentan su consumo; sin embargo, amoxicilina disminuía. También el estudio realizado en ocho países latinoamericanos ${ }^{15}$ señala que en todos ellos aumentó el consumo de quinolonas entre los años 1997 y 2007.

El estudio señalado menciona, respecto de Chile, que las medidas de corto plazo tuvieron gran impacto inicial, que duró aproximadamente dos años, pero desafortunadamente, el consumo de antimicrobianos comenzó a aumentar desde 2002, retornando a niveles semejantes a 1997. La Figura 2 muestra esta tendencia al aumento, que seguirá en ascenso si no se toman las medidas a largo plazo necesarias a nivel país para evitarlo.

El aumento de consumo de amoxicilina, aminopenici- 
linas con inhibidores de $\beta$-lactamasas, macrólidos como azitromicina y claritromicina y quinolonas documentado en este estudio, coincide con otros siete países latinoamericanos ${ }^{15}$. Incluso, en Venezuela, las medidas regulatorias que se implementaron no mostraron impacto en el consumo. Esto destaca el rol de los prescriptores y su necesidad de abordaje comomedida complementaria a futuro.

La globalización y migración de personas entre países crea nuevas posibilidades de trasmisión de resistencia antimicrobiana ${ }^{4}$ y los países deben saber responder a esta problemática ecológica, por lo cual deberían incentivarse sistemas de vigilancia de consumo en países como el nuestro, donde médicos prescriben antimicrobianos en forma irracional, donde las personas presionan al médico para la prescripción de antimicrobianos, donde es factible comprarlos sin prescripción médica y las autoridades de salud no tienen políticas ni asignan recursos para evitar estas situaciones. Se debe considerar, además, el gasto involucrado en el consumo de antimicrobianos. La Tabla 5 muestra un gasto de 45 millones de dólares en antimicrobianos vendidos en farmacias privadas, durante 1998 y el valor se repite el año 2008 .

\section{Conclusiones}

- Las medidas regulatorias impuestas por el Ministerio de Salud de Chile en 1999, tuvieron un impacto inicial; sin embargo, no hubo nuevas medidas ni reforzamiento en el tiempo, no hubo control de cumplimiento de estas medidas regulatorias, todo lo cual favoreció que el impacto no perdurara en el tiempo.

- Estas medidas regulatorias no afectaron el grupo de quinolonas y macrólidos nuevos y su aumento de consumo ha sido progresivo resaltando en estos grupos el rol de los médicos prescriptores.
- Las DDD/1.000 habitantes-día es una unidad fácil de usar, de bajo costo, que permite efectuar comparaciones, a pesar de sus limitaciones.

- Velar por el uso racional de los antimicrobianos es una tarea que debe ser permanente y requiere apoyo de las autoridades de salud para capacitar a los prescriptores además de educar a la población en general, sobre las implicancias del uso inadecuado de antimicrobianos y su efecto sobre la ecología microbiana.

\section{Resumen}

Introducción: El Ministerio de Salud de Chile reguló el consumo de antimicrobianos en el mes de septiembre de 1999, mediante restricción de venta limitada sólo a venta con receta médica. El objetivo de este estudio es conocer el impacto de las medidas regulatorias entre los años 2000 y 2008. Metodología: Se realizó un análisis retrospectivo de las ventas en farmacias privadas desde el año 2000 al 2008. La información se obtuvo del International Marketing System Health (IMS Health), una empresa internacional encargada de auditar las ventas de farmacias. La unidad de consumo usada fue la DDD/1.000 habitantes-día. Resultados: A partir del año 2000 al 2002, las medidas regulatorias tuvieron un gran impacto; sin embargo, desde el año 2002 el consumo de antimicrobianos aumentó, especialmente amoxicilina, obteniéndose valores similares a los existentes en 1998. Conclusiones: Las medidas regulatorias tuvieron un impacto inicial; sin embargo, no fueron reforzadas ni controladas en el tiempo. Es necesario que las autoridades de salud del país asuman la tarea permanente de educar a la comunidad acerca del uso inadecuado de antimicrobianos y su efecto sobre la ecología bacteriana.

\section{Referencias}

1.- Bavestrello L, Cabello A. Estudio del consumo de antimicrobianos en la comunidad. Chile, diez años después. Rev Chil Infect 1999; 16: 185-90.

2.- Bavestrello L, Cabello A. Tendencia del consumo de antimicrobianos en Chile. En: R. Salvatierra-González, Yehuda Benguigui. Resistencia antimicrobiana en las Américas: Magnitud del problema y su contención. Organización Panamericana de la Salud 2000; 234-40.

3.- Bavestrello L, Cabello A, Casanova D. Impacto de medidas regulatorias en la tendencia de consumo comunitario de antibióticos en Chile. Rev Med Chile 2002; 130: 1265-72.

4.- Harbarth S, Samore M. Antimicrobials resistance determinants and future control. Emerg Infect Dis 2005; 11: 794-801.
5.- García P. Resistencia bacteriana en Chile. Rev Chil Infect 2003; 20 suppl 1: 11-23.

6.- Vecchiola M. Bichos resistentes a los antimicrobianos en el siglo 21. Rev Chil Infect 2009; 26: 568-9.

7.- Second International Conference on improving use of medicines. ICIUM 2004. Abstract AM003, oral presentation.Thailand. http:// archives.who.int/icium/icium2004 acceso 03 diciembre 2009.

8.- Bavestrello L, Cabello A. How Chile tackled overuse of antimicrobials. WHO Essential Drug Monitor 2000; 28-29: 13-14.

9.- Pharmaceutical Market. Chile. International Market Statistics, IMS Health Chile Ltda. 20002008. http://www.imshealth.com/portal/site/ imshealth (acceso 03 enero 2010).

10.- Monnet D. Measuring antimicrobial use: The way forward. Clin Infect Dis 2007; 44 : 671-3.
11.- WHO Collaborating Centre for Drug Statistics and Methodology. ATC/DDD system. Available at: http//www.whocc.no

12.- INE Instituto Nacional de Estadísticas de Chile. :http//www.ine.cl/canales/chile Ver Proyecciones y estimaciones de población total país, 1950-2050 (acceso 14 octubre 2009).

13.- Petersen I, Hayward A, Antibacterial prescribing in primary care. SACAR Surveillance Subgroup. J Antimicrob Chemother 2007; 60 Suppl 1: i43-i47

14.- McCaig L, Besser R, Hughes J. Antimicrobial drug prescription in ambulatory care settings, United States, 1992-2000. Emerg Infect Dis 2003; 9: $432-7$.

15.- Wirtz VJ, Dreser A, Gonzales R. Trend in antibiotic utilization in eight Latin American countries, 1997-2007. Rev Panam Salud Pública 2010; $27: 219-25$. 